\title{
Nutrición enteral total $v s$. nutrición parenteral total en pacientes con pancreatitis aguda grave
}

\author{
M. Casas, J. Mora ${ }^{1}$, E. Fort ${ }^{2}$, C. Aracil, D. Busquets, S. Galter, C. E. Jáuregui, E. Ayala, D. Cardona ${ }^{3}$, \\ I. Gich ${ }^{4}$ y A. Farré \\ Servicios de Patología Digestiva y 'Bioquímica. Hospital de la Santa Creu i Sant Pau. Barcelona. ${ }^{2}$ Servicio de Aparato \\ Digestivo. Hospital Universitari Dr. Josep Trueta. Girona. Servicios de ${ }^{3}$ Farmacia y ${ }^{4}$ Epidemiología Clínica $i$ Salut \\ Pública. Hospital de la Santa Creu i Sant Pau. Barcelona
}

\section{RESUMEN}

Objetivo: comparar la eficacia de la instauración precoz de nutrición enteral total (NET) frente a nutrición parenteral total (NPT) en pacientes con pancreatitis aguda grave (PAG).

Métodos: estudio prospectivo aleatorio. Se incluyeron consecutivamente 22 pacientes con PAG aplicando los criterios APACHE II, valores de PCR y graduación de Balthazar en la TC. El grupo I $(n=11)$ recibió NPT y el grupo II $(n=12)$ NET. Se valoró la respuesta inflamatoria (PCR, TNF- $\alpha$, IL-6), las proteínas viscerales (pre-albúmina, albúmina), la tasa de complicaciones (síndrome de respuesta inflamatoria sistémica, fallo multiorgánico, infecciones), las intervenciones quirúrgicas, la estancia hospitalaria y la mortalidad.

Resultados: no hubo diferencias significativas en los primeros 10 días entre los dos grupos en la evolución de los criterios APACHE II, en las concentraciones de PCR, TNF- $\alpha$ e IL- 6 ni tampoco en los valores de pre-albúmina y albúmina. Siete pacientes del grupo I presentaron complicaciones graves frente a 4 del grupo II. Requirieron intervención quirúrgica 3 pacientes del grupo I. La estancia hospitalaria fue similar en los dos grupos. Dos pacientes del grupo I fallecieron.

Conclusiones: se ha observado una tendencia a una mejor evolución de los pacientes con PAG que utilizaron NET frente a los que utilizaron NPT.

Palabras clave: Pancreatitis aguda grave. Nutrición. PCR. TNF- $\alpha$. IL-6.

\begin{abstract}
Objective: to compare the efficacy of early total enteral nutrition (TEN) vs. total parenteral nutrition (TPN) in patients with severe acute pancreatitis (SAP).

Methods: a total of 22 consecutive patients with SAP were randomized to receive TPN (group I) or TEN (group II). SAP was defined applying APACHE II score, C-reactive protein (CRP) measurements and/or Balthazar CT scan score. Acute inflammatory response (CRP, TNF- $\alpha$, IL-6), visceral proteins (pre-albumin, albumin), complications (systemic inflammatory response syndrome, multiorgan failure, infections), surgical interventions, length of hospital stay and mortality were evaluated.

Results: no significant differences were found between the two groups in the APACHE II score, in CRP, TNF- $\alpha$ and IL-6 concentrations or in pre-albumin and albumin levels over the first 10 days. Seven patients in group I and 4 in group II suffered severe complications. Three patients in group I required surgical intervention. Length of hospital stay was alike in the two groups. Two patients from group I died in the course of the hospitalization.

Conclusions: SAP patients with TEN feeding showed a tendency towards a better outcome than patients receiving TPN.
\end{abstract}

Key words: Severe acute pancreatitis. Nutrition. C-reactive protein. TNF- $\alpha$. IL-6.

Casas M, Mora J, Fort E, Aracil C, Busquets D, Galter S, Jáuregui CE, Ayala E, Cardona D, Gich I, Farré A. Nutrición enteral total us. Nutrición parenteral total en pacientes con pancreatitis aguda grave. Rev Esp Enferm Dig 2007; 99: $264-269$.

Estudio realizado en parte con una ayuda del Instituto de Salud Carlos III. Ministerio de Sanidad y Consumo. Redes de Investigación Cooperativa (C03/02).

Recibido: 24-07-06.

Aceptado: 09-01-07.

Correspondencia: Antoni Farré Viladrich. Servicio de Patología Digestiva. Hospital de la Santa Creu i Sant Pau. Avda. Sant Antoni M ${ }^{\mathbf{a}}$ Claret, 167. 08025 Barcelona. e-mail: afarre@santpau.es

\section{INTRODUCCIÓN}

La pancreatitis aguda grave (PAG) es un proceso hipermetabólico e hiperdinámico que crea un estado de estrés catabólico acompañado de una respuesta inflamatoria y de un deterioro nutritivo en los pacientes que sufren este proceso (1).

La PAG, según la clasificación de Atlanta de 1992 (2), está reconocida como una enfermedad que puede cursar en dos fases (3), una inicial (estéril) en la que se podría 
desencadenar un síndrome de respuesta inflamatoria sistémica (SRIS) (4) con posibilidad de complicarse con una sepsis (5) y un fallo multiorgánico (FMO) (6) y ser la causa del fallecimiento del paciente en los primeros días y una segunda fase, generalmente a partir de la segunda semana de evolución, que puede acompañarse de complicaciones locales como la necrosis pancreática, que en el caso de sufrir una infección podría también ser letal para el paciente (2). La mayoría de las complicaciones y de los fallecimientos que ocurren en la PAG son consecuencia de la respuesta inmune e inflamatoria a la necrosis pancreática y/o a la infección.

Se tienen cada vez más evidencias de que el tubo digestivo desempeña un papel importante en la respuesta inmune e inflamatoria en la PAG. Datos experimentales sugieren que las citocinas endógenas responsables de esta respuesta son estimuladas por la endotoxina y otros productos bacterianos absorbidos por un intestino metabólicamente alterado (7). En este sentido, diversos estudios han demostrado en pacientes politraumatizados y en grandes quemados que el establecimiento de una nutrición enteral total (NET) disminuye significativamente la fase de respuesta inflamatoria aguda y la incidencia de complicaciones sépticas si se compara con la evolución de los que reciben una nutrición parenteral total (NPT) $(8,9)$. El mecanismo sugerido sería que la alimentación a través del tubo digestivo mantendría la función de la barrera intestinal impidiendo la translocación de las bacterias y de las toxinas presentes en la luz intestinal.

La NPT ha sido durante mucho tiempo la vía de alimentación utilizada con preferencia en los pacientes con PAG con objeto de mejorar el estado de nutrición del paciente y mantener en reposo al páncreas evitando el estímulo de su secreción. Sin embargo, la NPT tiene ciertos inconvenientes como son un incremento del riesgo de padecer infecciones del catéter colocado en la vía central, de hiperglicemias importantes, de alteraciones electrolíticas, etc., al mismo tiempo que la eliminación de la vía oral de alimentación favorece la alteración de la barrera intestinal con un aumento de su permeabilidad $(10,11)$.

En los últimos años son varios los estudios prospectivos aleatorizados (12-19), entre los que está incluido un meta-análisis (15), en donde se demuestran las ventajas de la NET administrada de forma precoz en los pacientes con pancreatitis aguda, como medida terapéutica para la atenuación del SRIS y de las complicaciones sépticas. Los beneficios potenciales serían el mantenimiento de la barrera mucosa intestinal que impediría la translocación bacteriana y por otra parte la eliminación de las infecciones asociadas a la NPT. Sin embargo, entre los estudios anteriormente mencionados escasean los que únicamente hayan incluido a pacientes con PAG $(13,18,19)$. El objetivo de este trabajo fue comparar la eficacia de la instauración precoz de NET $v s$. NPT en la evolución de la PAG en nuestro medio.

\section{MATERIAL Y MÉTODOS}

Este estudio trata de un ensayo clínico prospectivo aleatorio en el que se han incluido de forma consecutiva un total de 22 pacientes distribuidos en dos grupos según una tabla de números aleatorios previamente obtenida por ordenador. La opción correspondiente a cada paciente, grupos (I y II), estaba contenida en un sobre cerrado que se abría justo antes de la inclusión: los pacientes incluidos en el grupo I $(n=11)$ recibieron NPT y los incluidos en el grupo II $(n=11)$ recibieron NET.

Se incluyeron pacientes varones y mujeres de edad $\geq$ a 18 años que a su ingreso hospitalario fueron diagnosticados de PAG de cualquier etiología. Se consideraron criterios de pancreatitis aguda la clínica sugestiva (dolor abdominal, náuseas, vómitos, etc.) y la elevación de amilasa y/o lipasa sérica por encima de 3 veces el límite superior de la normalidad. Fueron considerados criterios de PAG los pacientes que dentro de las primeras 48 horas de inicio del proceso tuvieran dos o más de los siguientes hallazgos: una puntuación $\geq 8$ en el sistema de evaluación de gravedad Acute Physiology and Chronic Health Evaluation (APACHE II) (20), una PCR > $150 \mathrm{mg} / \mathrm{L}$ o una $\mathrm{TC}$ abdominal con afectación pancreática grado $\mathrm{D}$ o $\mathrm{E}$ de Balthazar (21). Fueron considerados criterios de exclusión la edad inferior a 18 años, el embarazo y las exacerbaciones de pancreatitis crónica.

Todos los pacientes fueron sometidos a control intensivo como mínimo durante las primeras 72 horas de su ingreso hospitalario. Durante la fase aguda recibieron una adecuada reposición hidroelectrolítica, se sometieron a una monitorización hemodinámica y a una asistencia en UCI de las funciones respiratoria y renal cuando fue preciso ( 7 pacientes del grupo I y 4 pacientes del grupo II). En este periodo, de un máximo de 72 horas, los pacientes fueron aleatorizados y distribuidos en uno de los dos grupos instaurándose de forma inmediata el tipo de nutrición correspondiente.

Los pacientes del grupo I, recibieron NPT mediante infusión continua durante las 24 horas a través de un catéter colocado por vía central (subclavia/yugular). La infusión se inició a una velocidad de $40 \mathrm{ml} / \mathrm{h}$ que se fue incrementando en $20 \mathrm{ml} / \mathrm{h}$ cada 4 horas hasta conseguir las necesidades requeridas por el paciente. El objetivo a conseguir era la administración de $1,5-2 \mathrm{~g}$ de proteínas $/ \mathrm{kg} /$ día y 30 $35 \mathrm{kcal} / \mathrm{kg} /$ día. La relación de calorías no proteicas/nitrógeno que se intentó conseguir fue de 100-125/1.

Los pacientes del grupo II, recibieron NET (PEPTISORB $^{\circledR}$, Nutricia S.R.L. Madrid) a través de una sonda nasoyeyunal de calibre $10 \mathrm{~F}$ y de una longitud de $114 \mathrm{~cm}$ colocada bajo control radiológico intentando que su extremo distal quedase situado en la zona del ángulo de Treitz. El ritmo de infusión fue de $25 \mathrm{ml} / \mathrm{h}$ con incrementos de $25 \mathrm{ml} / 4 \mathrm{~h}$ hasta conseguir las necesidades requeridas por el paciente. El objetivo en cuanto a necesidades nutritivas fue el mismo que en el grupo I (1,5-2 g de proteínas/kg/día y 30-35 kcal/kg/día). 
Durante los primeros 10 días, junto a los controles clínicos y bioquímicos habituales, se monitorizaron cada 48 horas las concentraciones de PCR, de las interleucinas proinflamatorias TNF- $\alpha$ e IL-6 y la determinación de proteínas viscerales (pre-albúmina y albúmina). El número total de muestras analizadas correspondientes a los dos grupos fue de 396. Los pacientes incluidos en el estudio fueron seguidos durante todo el periodo de hospitalización mediante control clínico del dolor, ritmo intestinal, trastornos metabólicos, cultivos de diferentes fluidos si aparecía fiebre y exploración mediante TC abdominal cuando se consideró necesario con el fin de detectar necrosis y la posible formación de abscesos. Las determinaciones en el suero de las concentraciones de TNF- $\alpha$ y de IL-6 se realizaron mediante dos inmunoanálisis enzimoquimioluminométricos en el analizador automático Immulite $^{\circledast}$ (Diagnostic Products Co., Los Angeles, CA, EE.UU.). Los valores de corte utilizados fueron $\leq 8 \mathrm{ng} / \mathrm{L}$ para TNF- $\alpha$ y $\leq 4 \mathrm{ng} / \mathrm{L}$ para IL-6, valores que fueron establecidos por el fabricante basados en controles sanos.

Como objetivos del estudio se valoraron: la respuesta inflamatoria (PCR, TNF- $\alpha$, IL-6), la determinación de proteínas viscerales (pre-albúmina, albúmina), la tasa de complicaciones (SRIS, FMO, infecciones), las intervenciones quirúrgicas a que fueron sometidos, la estancia hospitalaria y la mortalidad.

El análisis estadístico se realizó a través del test de Mann-Whitney, el test exacto de Fisher y el test de $\chi^{2}$ con un intervalo de confianza del $95 \%$.

Este estudio fue autorizado por el Comité de Ética y Ensayos Clínicos del propio hospital y se obtuvo el consentimiento informado de todos los pacientes introducidos.

\section{RESULTADOS}

Los datos demográficos incluyendo la etiología quedan reflejados en la tabla I.

No se observaron diferencias significativas entre los dos grupos en cuanto a la gravedad inicial de la pancreatitis valorada a través de la aplicación de los criterios de

Tabla I. Datos demográficos y etiología de la pancreatitis

\begin{tabular}{lll}
\hline Variable & \multicolumn{1}{c}{$\begin{array}{c}\text { Grupo / (NPT) } \\
n=11 p .\end{array}$} & \multicolumn{1}{c}{$\begin{array}{c}\text { Grupo II (NET) } \\
n=11 \mathrm{p} .\end{array}$} \\
\hline Edad (años) & $55,6 \pm 15,6$ & $61,2 \pm 16,6$ \\
Sexo & 8 varones & 8 varones \\
& 3 mujeres & 3 mujeres \\
Etiología & 7 biliar & 4 biliar \\
& 4 alcohólica & 1 alcohólica \\
& & 3 otras etiologías \\
& & 3 idiopática
\end{tabular}

De los pacientes del grupo II con otras etiologías, 2 correspondieron a origen farmacológico y 1 a hipertrigliceridemia. gravedad APACHE II y de las determinaciones de PCR. Respecto a la gravedad determinada mediante la TC a través de la graduación de Balthazar hubo 5 pacientes con grado D-E en el grupo I y 3 pacientes en el grupo II.

No hubo complicaciones relacionadas con la colocación de la sonda de alimentación y por otra parte la administración de NET fue bien tolerada en todos los pacientes que la recibieron si bien fue necesario reducir la velocidad de infusión en la mayoría de ellos durante los primeros 4 días debido a diversos problemas (náuseas, sensación de plenitud, diarrea, etc.). Al cabo de 5 días la totalidad de pacientes sometidos a NET recibieron la cantidad de calorías deseada (Tabla II). La sonda de alimentación permaneció colocada un mínimo de 15 días en la mayoría de pacientes. En el transcurso de la enfermedad, 2 pacientes del grupo I presentaron infección de la vía central confirmada por la obtención de cultivo positivo para germen patógeno de la punta del catéter. Otros 3 pacientes de este grupo presentaron hemocultivos positivos: 1 para Stafilococo epidermidis y Pseudomonas, 1 para Stafilococo aureus y 1 para Streptococo agalactiae.

Tabla II. Calorías y nitrógeno recibidos por los dos grupos

\begin{tabular}{lccc}
\hline \multicolumn{1}{c}{ Variable } & $\begin{array}{c}\text { Grupo I (NPT) } \\
n=11 p .\end{array}$ & $\begin{array}{c}\text { Grupo II (NET) } \\
n=11 p .\end{array}$ \\
\hline Calorías/kg/día & $20,8 \pm 1,68$ & $(p=n s)$ & $20,09 \pm 1,83$ \\
Nitrógeno & $0,186 \pm 0,009$ & $(p<0,005)$ & $0,148 \pm 0,016$ \\
(g/kg/día) & & &
\end{tabular}

Datos obtenidos a partir del $5^{\circ}$ día en que los pacientes del grupo II alcanzaron la cantidad de calorías deseada. El periodo mínimo de estudio con ambos tipos de nutrición fue de 10 días.

No hubo diferencias significativas entre los dos grupos, durante los 10 primeros días del curso de la enfermedad, en la evolución de los criterios APACHE II, en las concentraciones de PCR, TNF- $\alpha$ e IL-6 (Figs. 1 y 2) ni tampoco en los valores de pre-albúmina y albúmina (Fig. 3).

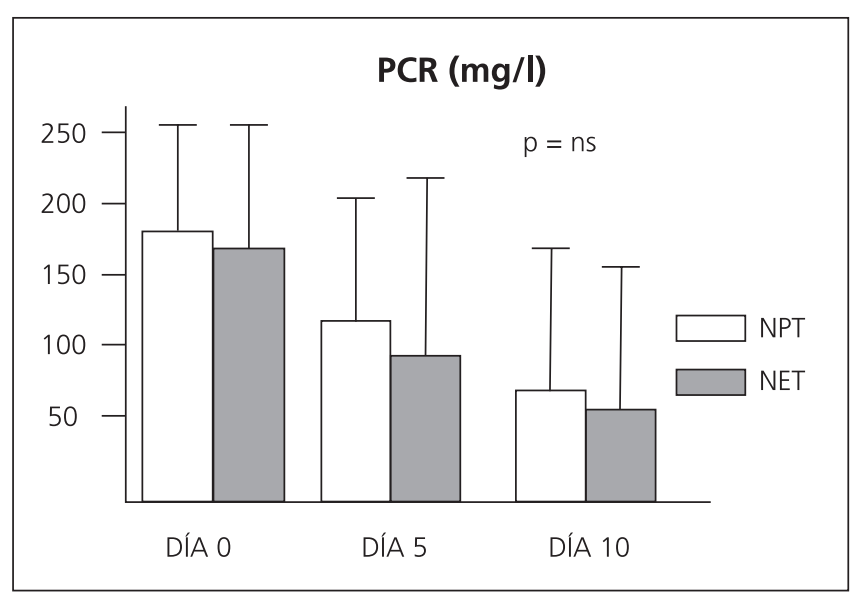

Fig. 1. Concentraciones de PCR iniciales, a los 5 y a los 10 días de evolución de la enfermedad según la nutrición recibida. 


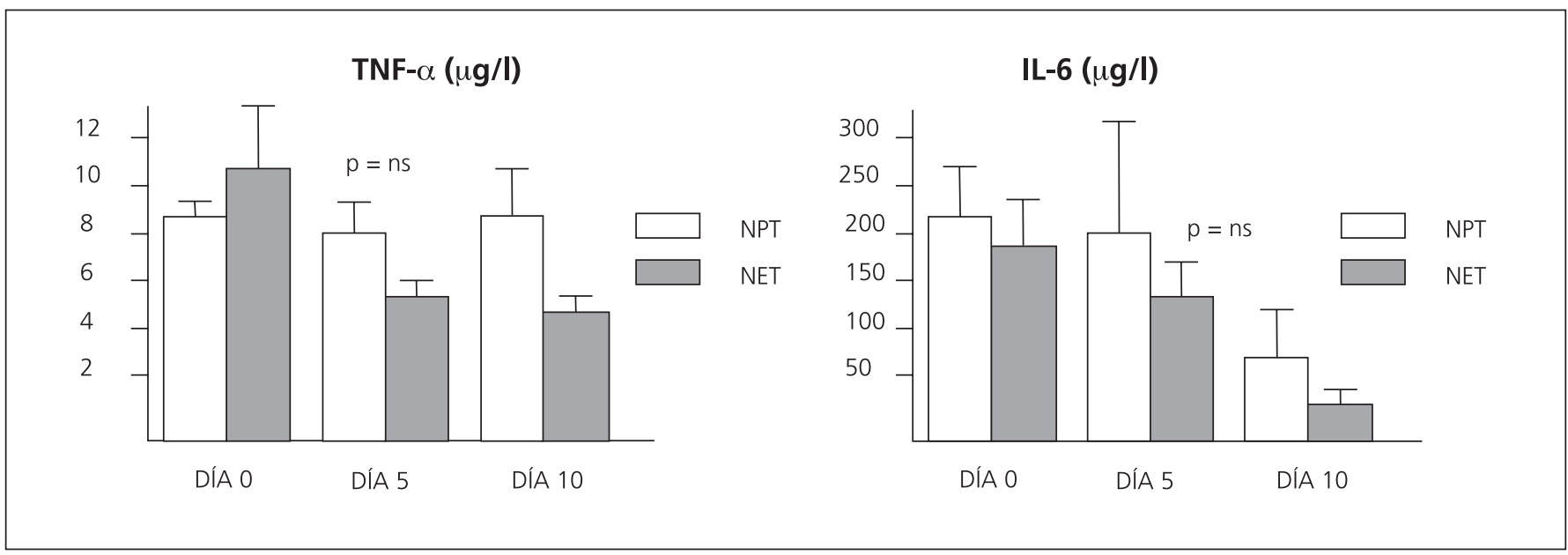

Fig. 2. Concentraciones de TNF- $\alpha$ e IL-6 iniciales, a los 5 y a los 10 días de evolución de la enfermedad según la nutrición recibida.

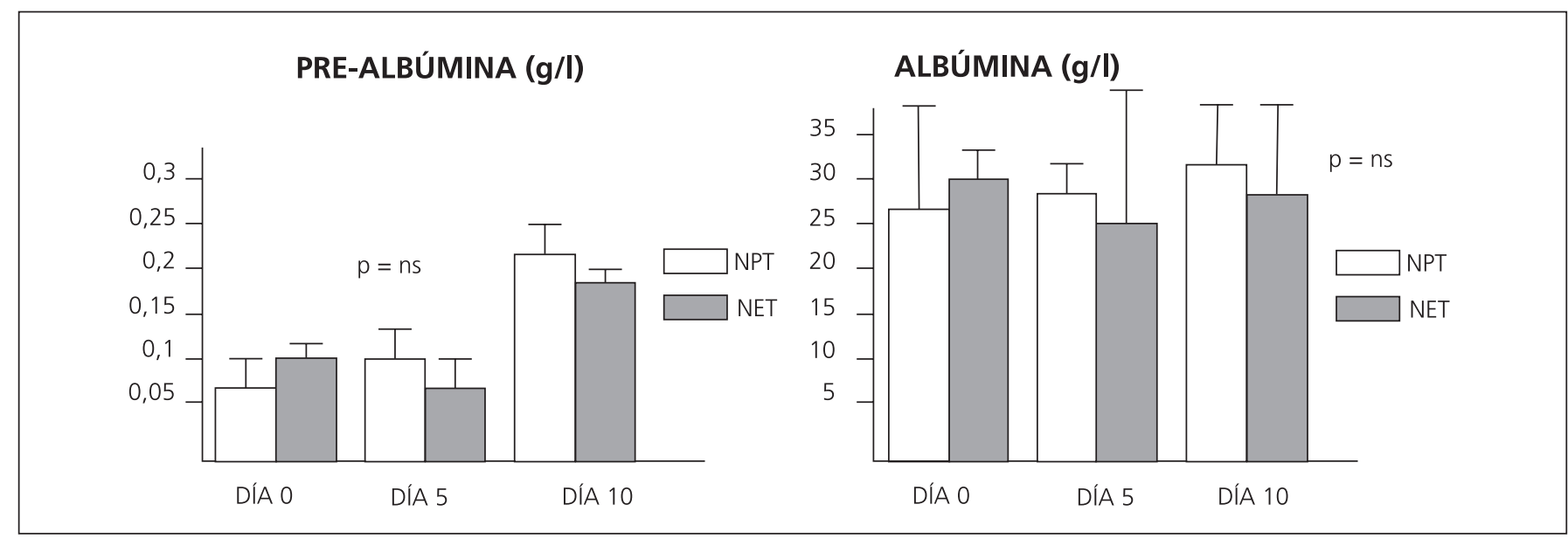

Fig. 3. Concentraciones de pre-albúmina y albúmina iniciales, a los 5 y a los 10 días de evolución de la enfermedad según la nutrición recibida.

Siete pacientes del grupo I, tuvieron complicaciones graves; 3 pacientes presentaron infección ( 2 de ellos necrosis infectada), 2 pacientes FMO (1 de ellos con infección) y 2 pacientes SRIS (ambos con infección). En el grupo II, 4 pacientes presentaron complicaciones graves, 2 de ellos SRIS, 1 infección del tracto urinario y 1 trombosis portal (Tabla III). Once pacientes, 6 del grupo I y 5

Tabla III. Complicaciones de la PAG en los dos grupos

\begin{tabular}{lcc}
\hline Complicación & $\begin{array}{c}\text { Grupo I (NPT) } \\
n=11 p .\end{array}$ & $\begin{array}{c}\text { Grupo II (NET) } \\
n=11 p .\end{array}$ \\
\hline Infección & 5 & 1 \\
FMO & 2 & - \\
SRIS & 2 & 2 \\
Necrosis & 2 & - \\
Otras & - & 1 \\
\hline
\end{tabular}

Algunos pacientes presentaron varias complicaciones simultáneas (ver texto). del grupo II, recibieron antibióticos. Todos los pacientes del grupo II fueron tratados con imipenem, mientras que en el grupo I sólo 2 de ellos fueron tratados con este antibiótico en tanto que los 4 restantes fueron tratados con piperacilina/tazobactam.

Tres pacientes del grupo I requirieron intervención quirúrgica durante el ingreso hospitalario: 1 por FMO y necrosis pancreática, 1 por colección infectada y drenaje de colecciones en una primera intervención y para desbridamiento de necrosis y drenaje de colecciones en una segunda intervención y por último 1 paciente fue sometido a drenaje de un absceso pancreático (Tabla IV).

La estancia media hospitalaria fue similar en ambos grupos (Tabla IV). Dos pacientes del grupo I y 1 del grupo II requirieron estancia en UCI.

Finalmente, 2 pacientes del grupo I fallecieron: 1 a consecuencia de necrosis pancreática y FMO y el otro por broncoaspiración en el postoperatorio de drenaje quirúrgico de colecciones peripancreáticas (Tabla IV). 


\begin{tabular}{ccc}
$\begin{array}{c}\text { Tabla IV. Datos correspondientes a intervenciones } \\
\text { quirúrgicas, estancia media hospitalaria y mortalidad }\end{array}$ \\
\hline Variable & $\begin{array}{c}\text { Grupo / (NPT) } \\
n=11 \mathrm{p} .\end{array}$ & $\begin{array}{c}\text { Grupo II (NET) } \\
n=11 \mathrm{p} .\end{array}$ \\
\hline Interv. quirúrgica & $\begin{array}{c}3 \text { (1 necrosis y FMO, } \\
1 \text { necrosis y colecciones, } \\
1 \text { absceso) } \\
\text { Estancia media }\end{array}$ & - \\
hospitalaria (días) & 30,7 \\
Exitus & $\begin{array}{c}2 \text { (1 necrosis y FMO, } \\
\text { broncoaspiración } \\
\text { postoperatoria) }\end{array}$ \\
\hline
\end{tabular}

\section{DISCUSIÓN}

El tratamiento de la PAG está basado en medidas de soporte entre las cuales mantener unos niveles de nutrición adecuados es un objetivo esencial. Estudios realizados en animales y en humanos han mostrado que la NPT puede deteriorar la inmunidad intestinal y sistémica y contribuir a la atrofia de la mucosa del intestino con el consiguiente deterioro en la función de barrera intestinal (22-25).

Hasta el momento son escasos los estudios prospectivos randomizados con criterios de rigor científico que comparen la NET vs. la NPT en el curso de una pancreatitis aguda (15). Por otra parte, debe destacarse que tampoco abundan los estudios que incluyan únicamente a pacientes con PAG tal como se ha efectuado en el presente trabajo $(13,19)$. Aunque reconocemos que este es un estudio con un número limitado de pacientes los resultados obtenidos son consistentes con respecto a los alcanzados en otros trabajos realizados en pacientes con PAG $(13,14,26)$.

En este estudio no surgieron dificultades en la colocación de la sonda ni en su mantenimiento en la posición correcta. Al igual que en otras series (27), la NET estuvo generalmente bien tolerada en los pacientes con íleo paralítico. Sin embargo, en los casos en los que no se puedan administrar las calorías necesarias o mantener la sonda de alimentación en el lugar requerido puede hacerse precisa la instauración de NPT (28); esta circunstancia no ocurrió en ningún paciente de esta serie. La reciente publicación de un trabajo en el que no se observaron diferencias clínicas entre un grupo de pacientes que recibió NET a través de una sonda nasogástrica con respecto a un grupo que la recibió a través de sonda naso-yeyunal abre nuevas posibilidades que merecen ser confirmadas (29).

En este trabajo no se han observado diferencias significativas entre NET y NPT, en la evolución de los marcadores de inflamación (PCR, TNF- $\alpha$ e IL-6) durante los primeros 10 días de la enfermedad ni en los criterios APACHE II. Estos resultados no difieren de los obtenidos por Powell y cols. (26) en un grupo randomizado de 28 pacientes con PAG. Sin embargo, en otros trabajos se ha descrito una mejora de la respuesta inflamatoria sisté- mica, así Windsor y cols. (14) destacan una reducción significativa de los valores de PCR en los pacientes que fueron sometidos a NET con la salvedad de que las concentraciones iniciales de PCR fueron menores que los observados en nuestro estudio. Por su parte, McClave y cols. (12), observaron una reducción en la puntuación inicial de los criterios de gravedad (Ranson, APACHE III) en el grupo de pacientes que recibieron NET, en el que también estaban incluidas formas leves o moderadas de pancreatitis aguda. Un incremento de la respuesta inflamatoria sistémica, puesta de manifiesto por la elevación de citocinas proinflamatorias (IL-6, etc.) en pacientes sometidos a NPT, ha sido también descrita en otras situaciones de estrés hipermetabólico (30,31).

Respecto a las complicaciones que aparecieron en los pacientes con PAG de esta serie, se ha observado su predominio en el grupo que recibió NPT con presencia de necrosis pancreática, colecciones, FMO y SRIS a las que con frecuencia se sobreañadieron infecciones por diversos gérmenes. Esta observación concuerda con la mayoría de estudios realizados en los que se detecta, en contraposición a los pacientes que recibieron NPT, una significativa reducción de las infecciones en el grupo de los que recibieron NET (15). Así Windsor y cols. (14) describen 3 episodios de sepsis en 18 pacientes sometidos a NPT contra ninguno en el grupo de pacientes con NET mientras que Kalfarentzos y cols. (13) detallan 4 pacientes con necrosis infectada o necrosis peripancreática en el grupo de 20 pacientes con NPT frente a 1 solo paciente en el grupo de 18 pacientes con NET. Es destacable el haberse puesto de manifiesto en otras situaciones clínicas extradigestivas que la NET también limita el número de complicaciones sépticas en comparación con la NPT $(32,33)$. Por otra parte, aunque no se ha logrado demostrar de forma fehaciente, el hecho de que la mayoría de microorganismos responsables de las infecciones en las PAG sean gérmenes entéricos patógenos sugiere que la translocación bacteriana podría ser el mecanismo responsable de la patogénesis de las sepsis en la pancreatitis (34-36). En lo que respecta a las complicaciones no sépticas no hemos logrado demostrar diferencias notables en su incidencia, según el tipo de nutrición recibida, lo cual concuerda con lo descrito por otros autores (13-18).

En el presente estudio, la intervención quirúrgica fue únicamente necesaria en pacientes que recibieron NPT. Cuatro estudios incluidos en el meta-análisis realizado por Marik y cols. (15) demuestran una reducción significativa de intervenciones quirúrgicas en los grupos sometidos a NET probablemente relacionado con la menor tasa de complicaciones sépticas que sufren estos pacientes. De forma similar, este mismo meta-análisis (15) destaca una reducción significativa de la estancia media hospitalaria en los pacientes del grupo que utilizó la vía enteral para su nutrición lo cual difiere de lo ocurrido en nuestra serie en la que esta variable tuvo un comportamiento similar en los dos grupos. Es probable que la heterogeneidad en la gravedad de los pacientes incluidos en 
los estudios anteriormente mencionados pueda explicar esta disparidad de resultados dado que en nuestro estudio únicamente se incluyeron a pacientes con PAG.

Por último, aunque en el presente estudio los dos únicos pacientes que fallecieron estaban incluidos en el grupo NPT, en la mayoría de trabajos publicados no se ha logrado detectar diferencias significativas en la mortalidad según el tipo de nutrición establecida (15).

De forma similar a las conclusiones alcanzadas en la mayoría de series publicadas, el presente estudio permite también vislumbrar una tendencia a una mejor evolución de los pacientes con PAG que utilizaron la vía enteral de alimentación frente a los que utilizaron la vía parenteral.

\section{AGRADECIMIENTOS}

Los autores quieren expresar su agradecimiento a Raquel Boluda por su ayuda técnica en las determinaciones analíticas y a Carolyn Newey por su soporte en la elaboración del manuscrito.

\section{BIBLIOGRAFÍA}

1. Havala T, Shronts E, Cerra F. Nutritional support in acute pancreatitis. Gastroenterol Clin North Am 1989; 18: 525-42.

2. Bradley E. A clinically based classification system for acute pancreatitis:Summary of the Atlanta syposium. Arch Surg 1993; 128: 58690.

3. Winslet MC, Hall C, London NJ, Neoptolemos JP. Relation of diagnostic serum amylase levels to aetiology and severity of acute pancreatitis. Gut 1992; 33: 982-6.

4. Nyström P-O. The systemic inflamatory response syndrome: definitions and aetiology. J Antimicrob Chemother 1998; 41 (Supl A): 1-7.

5. Wilson PG, Manji M, Neoptolemos JP. Acute pancreatitis as a model of sepsis. J Antimicrob Chemother 1998; 41 (Supl A): 51-63.

6. Poves Prim I, Fabregat Pous J, García Borobia FJ, Jorba Martí R, Figueras Felip J, Jaurrieta Mas E. Early onset organ failure is the best predictor of mortality in acute pancreatitis. Rev Esp Enferm Dig 2004; 96: 705-9.

7. Martínez J, Muñoz C, López M, Sánchez-Payá J, Laveda R, PérezMateo M. Endotoxin and anti-endotoxin antibodies in the prognosis of acute pancreatitis. Rev Esp Enferm Dig 2002; 94: 406-16.

8. Moore EE, Jones TN. Benefits of immediate jejunostomy feeding after major abdominal trauma - a prospective randomized trial. J Trauma 1986; 26: 874-81.

9. Alexander JW, MacMillan BG, Stinnert JD. Beneficial effects of aggresive protein feeding in severely burned children. Surgery 1980; 192: 505-18.

10. Grant JG, James S, Gratsowski V, Trexler KM. Total parenteral nutrition in pancreatic disease. Ann Surg 1984; 200: 627-31.

11. Buchman AL, Moukarzel AA, Bhuta S, Belle M, Ament ME, Eckhert $\mathrm{CD}$, et al. Parenteral nutrition is associated with intestinal morphologic and functional changes in humans. JPEN J Parenter Enteral Nutr 1995; 19: 453-60.

12. McClave SA, Greene LM, Snider HL, Makk LJK, Cheadle WG, Owens NA, et al. Comparison of the safety of early enteral vs parenteral nutrition in mild acute pancreatitis. JPEN J Parenter Enteral Nutr 1997; 21: 14-20.

13. Kalfarentzos F, Kehagias J, Mead N, Kokkinis K, Gogos CA. Enteral nutrition is superior to parenteral nutrition in severe acute pancreatitis: results of a randomized prospective trial. Br J Surg 1997; 84: 1665-9.

14. Windsor ACJ, Kanwar S, Li AGK, Barnes E, Guthrie JA, Spark JI, et al. Compared with parenteral nutrition, enteral feeding attenuates the acute phase response and improves disease severity in acute pancreatitis. Gut 1998; 42: 431-5.

15. Marik PE, Zaloga GP. Meta-analysis of parenteral nutrition versus enteral nutrition in patients with acute pancreatitis. BMJ 2004; 328: 1407.

16. Olah A, Pardavi G, Belagyi T, Nagy A Issekutz A, Mohamed GE Early nasojejunal feeding in acute pancreatitis is associated with a lower complication rate. Nutrition 2002; 18: 259-62.

17. Abou-Assi S, Craig K, O'Keefe SJ. Hypocaloric jejunal feeding is better than total parenteral nutrition in acute pancreatitis: results of a randomised comparative study. Am J Gastroenterol 2002; 97: 2255-62.

18. Gupta R, Patel K, Calder PC, Yaqoob P, Primrose JN, Johnson CD. A randomised clinical trial to assess the effect of total enteral and total parenteral nutrition support on metabolic, inflammatory and oxidative markers in patients with predicted severe acute pancreatitis (APACHE II $\geq 6$ ). Pancreatology 2003; 3: 406-13.

19. Louie BE, Noseworthy T, Hailey D, Gramlich LM, Jacobs P, Warnock GL. Enteral or parenteral nutrition for severe pancreatitis: a randomized controlled trial and health technology assessment. Can J Surg 2005; 48: 298-306.

20. Knaus WA, Draper EA, Wagner DP, Zimmeman JE. APACHE-II: severity of disease classification. Crit Care Med 1985; 13: 818-29.

21. Balthazar EJ, Robinson DL, Megibow AJ, Ranson JHC. Acute pancreatitis: value of CT in stablishing prognosis. Radiology 1990; 174: 331-6.

22. Alverdy J, Chi HS, Sheldon GF. The effect of parenteral nutrition on gastrointestinal immunity. The importance of enteral stimulation. Ann Surg 1985; 202: 681-4.

23. Illig KA, Ryan CK, Hardy DJ, Rhodes J, Locke W, Sax HC. Total parenteral nutrition-induced changes in gut mucosal function: Atrophy alone is not the issue. Surgery 1992; 112: 631-7.

24. Meyer J, Yurt RW, Duhaney R, Hesse DG, Tracey KJ, Fong Y, et al. Differential neutrophil activation before and after endotoxin infusion in enterally versus parenterally fed volunteers. Surg Gynecol Obstet 1988; 167: 501-9.

25. Groos S, Hunefeld G, Luciano L. Parenteral versus enteral nutrition: morphologic changes in human adult intestinal mucosa. J Submicrosc Cytol Pathol 1996; 28: 61-74.

26. Powell JJ, Murchison JT, Fearon KCH, Ros JA, Siriwardena AK. Randomized controlled trial of the effect of early enteral nutrition on markers of the immflamatory response in predicted severe acute pancreatitis. Br J Surg 2000; 87: 1375-81.

27. Meier R; Beglinger C, Layer P, Gullo L, Keim V, Laugier R, et al ESPEN guidelines on nutrition in acute pancreatitis. European Society of Parenteral and Enteral Nutrition. Clin Nutr 2002; 21: 173-83.

28. McClave SA, Dryden GW. Issues of nutritional support for the patient with acute pancreatitis. Semin Gastrointest Dis 2002; 13: 154-60.

29. Eatock FC, Chong P, Menezes N, Murray L, McKay CJ, Carter CR, et al. A randomized study of early nasogastric versus nasojejunal feeding in severe acute pancreatitis. Am J Gastroenterol 2005; 100: 432-9.

30. Gianotti L, Braga M, Vignali A, Balzano G, Zerbi A, Bisagni P, et al Effect of route of delivery and formulation of postoperative nutritional support in patients undergoing major operations for malignant neoplasms. Arch Surg 1997; 132: 1222-9.

31. Lin MT, Saito H, Fukushima R, Inaba T, Fukatsu K, Inoue T, et al Preoperative total parenteral nutrition influences postoperative systemic cytokine responses after colorectal surgery. Nutrition 1997; 13 : $8-12$.

32. Kompan L, Kremzar B, Gadzijev E, Prosek M. Effects of early enteral nutrition on intestinal permeability and the development of multiple organ failure after multiple injury. Intensive Care Med 1999; 25: 157-61.

33. Kudsk KA. Gut mucosal support-enteral nutrition as primary therapy after multiple system trauma. Gut 1994; 35 (1 Supl): S52-S4.

34. Runkel NS, Moody FG, Smith GS, Rodriguez LF, LaRocco MT, Miller TA. The role of the gut in the development of sepsis in acute pancreatitis. J Surg Res 1991; 51: 18-23.

35. Cicalese L, Sahai A, Sileri P, Rastellini C, Subbotin V, Ford H, et al Acute pancreatitis and bacterial translocation. Dig Dis Sci 2001; 46: 1127-32.

36. Nettelbladt CG, Katouli M, Bark T, Svenberg T, Molby R, Ljungqvist O. Evidence of bacterial translocation in fatal hemorragic pancreatitis. J Trauma 2000; 48: 314-5. 